\title{
Visual object pattern separation deficits in nondemented older adults
}

\author{
Chelsea K. Toner, ${ }^{1}$ Eva Pirogovsky, ${ }^{2}$ C. Brock Kirwan, ${ }^{3}$ and Paul E. Gilbert ${ }^{1,2,4}$ \\ ${ }^{1}$ Department of Psychology, San Diego State University, San Diego, California 92120, USA; ${ }^{2}$ San Diego State University/University \\ of California San Diego Joint Doctoral Program in Clinical Psychology, San Diego, California 92120, USA; ${ }^{3}$ Department of \\ Psychiatry, University of California, San Diego, California 92120, USA
}

\begin{abstract}
Young and nondemented older adults were tested on a continuous recognition memory task requiring visual pattern separation. During the task, some objects were repeated across trials and some objects, referred to as lures, were presented that were similar to previously presented objects. The lures resulted in increased interference and an increased need for pattern separation. For each object, the participant was asked to indicate whether (1) this was the first time the object was seen (new), (2) the object was seen previously (old), or (3) the object was similar to a previous object (similar). Older adults were able to correctly identify objects as old or new as well as young adults; however, older adults were impaired when identifying lures as similar. Therefore, pattern separation may be less efficient in older adults resulting in poorer recognition memory performance when interference is increased.
\end{abstract}

A primary region of the brain affected by normal aging is the hippocampus in humans (Good et al. 2001; Allen et al. 2005; Driscoll and Sutherland 2005; Raz et al. 2005; Walhovd et al. 2005) and animal models (Rosenzweig and Barnes 2003; Driscoll et al. 2006). However, the subregions of the hippocampus may be differentially affected by normal aging (Small et al. 2002, 2004). The dentate gyrus (DG) subregion may be particularly susceptible to age-related changes in both humans (Small et al. 2002) and animal models (Small et al. 2004; Patrylo and Williamson 2007), whereas aging may have less of an impact on pyramidal cells in the CA subregions (Small et al. 2002, 2004). In contrast, the CA subregions of the hippocampus may be more vulnerable to pathological aging associated with Alzheimer's disease (Braak and Braak 1996; Price et al. 2001; West et al. 2001). Therefore, tasks sensitive to dysfunction in particular subregions of the hippocampus may help to differentiate cognitive impairment associated with normal aging from pathological changes associated with Alzheimer's disease. Although hippocampal-dependent tasks are highly sensitive to age-related changes in the brain (for review, see Rosenzweig and Barnes 2003), behavioral studies in humans have not examined the performance of nondemented older adults on tasks shown to be particularly sensitive to DG function. As described in detail below, a key function of the DG may be to support pattern separation. Age-related changes in the DG may impair the ability to reduce similarity among new input patterns, resulting in decreased pattern separation (Wilson et al. 2006). Therefore, decreased efficiency in pattern separation may be a critical, but relatively unexamined, processing deficit in nondemented older humans.

Pattern separation is described as a mechanism for separating partially overlapping patterns of activation so that one pattern may be retrieved as separate from other patterns (Gilbert et al. 2001; Gilbert and Kesner 2006; Kirwan and Stark 2007). The operation of a pattern separation mechanism is critical for reducing potential interference among similar memory representations and increasing the likelihood of accurate encoding and

\footnotetext{
${ }^{4}$ Corresponding author.
}

E-mail pgilbert@sciences.sdsu.edu; fax (619) 594-3773.

Article is online at http://www.learnmem.org/cgi/doi/10.1101//m.1315109. subsequent retrieval. Models have suggested that the hippocampus may support pattern separation (Marr 1971; McNaughton and Nadel 1989; O'Reilly and McClelland 1994; Shapiro and Olton 1994; Rolls 1996, 2007; Rolls and Kesner 2006; Kesner 2007; Myers and Scharfman 2008). Based on characteristics of the mossy fiber system, pattern separation may be facilitated by the DG and its mossy fiber projections to the CA3 subregion of the hippocampus (Gilbert et al. 2001; Gilbert and Kesner 2006; Rolls and Kesner 2006; Leutgeb et al. 2007; McHugh et al. 2007; Rolls 2007; Bakker et al. 2008; Goodrich-Hunsaker et al. 2008; Hunsaker and Kesner 2008). Numerous studies have provided evidence that the DG and CA3 may play a key role in pattern separation using electrophysiological methods (Tanila 1999; Leutgeb et al. 2004, 2005, 2007; Leutgeb and Leutgeb 2007; Renaudineau et al. 2007), behavioral experiments utilizing localized neurotoxin induced lesions (Gilbert et al. 1998, 2001; Lee et al. 2005; Gilbert and Kesner 2006; Goodrich-Hunsaker et al. 2008; Hunsaker and Kesner 2008), and genetic manipulations (Cravens et al. 2006; Kubik et al. 2007; McHugh et al. 2007).

To examine pattern separation in humans, Kirwan and Stark (2007) developed a paradigm to measure brain activity using high resolution functional magnetic resonance imaging (fMRI) while young, healthy human participants performed a continuous recognition task involving highly similar stimuli. On this task, pictures of everyday objects were shown one at a time to each participant in the fMRI scanner. Some of the objects were repeated across trials and some objects, referred to as lures, were presented that were very similar but not identical to previously presented objects. The lures were hypothesized to result in increased interference and an increased need for pattern separation based on overlapping object features. Activity in the hippocampus distinguished between correctly identified old stimuli, correctly rejected similar lure stimuli, and false alarms to similar lures. The data provide compelling evidence that the hippocampus may play a significant role in pattern separation in humans. Another study from the same laboratory (Bakker et al. 2008) reported that when participants viewed these same stimuli in the fMRI scanner the results showed a consistent bias toward pattern separation in the DG and CA3 subregions (Bakker et al. 2008). Therefore, the DG and CA3 subregions of the human hippocampus may play a critical role in pattern separation. 
Despite substantial evidence suggesting that hippocampal dysfunction is associated with aging, studies have not examined pattern separation in nondemented older adults. Behavioral tasks that measure pattern separation may be a powerful and largely unexamined tool for the early detection of DG dysfunction associated with aging. Inefficient pattern separation may disrupt numerous mnemonic functions critical for the execution of daily living skills. Therefore, the present study examined pattern separation in nondemented older adults using a paradigm developed by Kirwan and Stark (2007).

The present study sample consisted of 20 nondemented older adults over $65 \mathrm{yr}$ of age (average $74.4 \mathrm{yr}$, SE 1.64) and 20 young adults (average $19.15 \mathrm{yr}$, SE 0.30 ). The average years of education was 13.85 (SE 0.26) years for young adults and 15.05 (SE 0.41) for older adults. A one-way analysis of variance revealed a statistically significant difference in education level $F_{(1,38)}=5.98, P<0.05$. However, the mean difference in education level was only one year and the higher education level was in the older adults. Older adults were screened for dementia using the Dementia Rating Scale (DRS) (Mattis 1976). The average DRS score for older adults was 136.10 (SE 1.02) and any participant who scored $<123$ was excluded from the study. Older adult participants also completed a battery of standardized neuropsychological tests including the Trail Making and Color-Word Interference subscales from the Delis Kaplan Executive Function System (D-KEFS) (Delis et al. 2001), as well as the Hopkins Verbal Learning Test-Revised (HVLTR) (Brandt 1991).

Young and older adults completed an established continuous recognition memory task requiring pattern separation for visual object information (Kirwan and Stark 2007; Bakker et al. 2008). Color photographs of everyday objects were shown one at a time on a computer screen to each participant. Some of the objects were repeated across trials and some objects, referred to as lures, were presented that were very similar but not identical to previously presented objects. The lures were hypothesized to result in increased interference and an increased need for pattern separation based on object features (Kirwan and Stark 2007). Participants were informed that several pictures of objects would be viewed during the task. When each object was presented, the participant was asked to press one of three buttons on a keyboard to indicate whether (1) this was the first time the object was seen during the task (new), (2) the object was seen previously during the task (old), or (3) the object was similar to an object seen previously during the task (similar). Instructions were visible at the bottom of the screen throughout testing. Participants were given six blocks of 108 stimuli each and were allowed to respond at their own pace. Trials were presented in random order with the mean lag separating the first and repeat or first and lure stimuli being $\sim 30$ trials.
The mean proportion of responses of old, similar, or new to first, repeated, and lure stimuli for young and older adults are shown in Figure 1. A $2 \times 3$ analysis of variance (ANOVA) with group (young adults, older adults) as a between-group factor and stimulus (first, repeat, lure) as a within-group factor was used to analyze correct responses. The analysis revealed a significant main effect of group $F_{(1,38)}=27.80, P<0.001$, a significant main effect of stimulus $F_{(2,76)}=85.73, P<0.001$, and a group $\times$ stimulus interaction $F_{(2,26)}=5.08, P<0.01$. A Newman-Keuls post-hoc comparison test of group $\times$ stimulus interaction revealed that young adults significantly outperformed older adults $(P<0.05)$ when correctly identifying lures as similar (Fig. 1). However, there were no significant age-related differences in correctly identifying first stimuli as new or repeated stimuli as old.

A $2 \times 2$ ANOVA with group (young adults, older adults) as a between-group factor and error type (old, new) as a within-group factor was used to analyze the types of errors committed in response to lure stimuli. The analysis revealed a significant main effect of group $F_{(1,38)}=15.50, P<0.001$ and a significant main effect of error type $F_{(1,38)}=101.58, P<0.001$; however, the group $\times$ stimulus interaction did not reach significance $F_{(1,38)}=2.11, P=$ 0.15 . The results demonstrate that nondemented older adults committed significantly more errors when identifying lure stimuli (Fig. 1).

To examine the number of correct responses to lure stimuli (responding similar) relative to the number of false positive responses to lures (responding old), difference scores were calculated for each participant by subtracting the number of old responses to lure stimuli from the number of similar responses to lure stimuli. A one-way ANOVA with group (young adults, older adults) as a between-group factor and the difference scores as the dependent variable revealed a significant between-group difference $F_{(1,38)}=14.57, P<0.001$. Younger adults were more likely to label a lure stimulus as similar, whereas older adults were more likely to label a lure as old (Fig. 2).

The present results indicate that young adults significantly outperformed older adults when correctly identifying lures as similar. Rather than correctly identifying lures as similar, older adults were more likely to commit false positive errors and identify the stimuli as old. Therefore, age-related changes in hippocampal subregions of nondemented older adults may result in less efficient pattern separation. However, there were no significant age-related differences in correctly identifying first stimuli as new or repeated stimuli as old. Therefore, the ability of older adults to correctly recognize repeated and new stimuli suggests that the agerelated differences in identifying lure stimuli were not due solely to general recognition memory deficits.

As mentioned previously, there is growing evidence suggesting that pattern separation is facilitated by the DG and its mossy
A

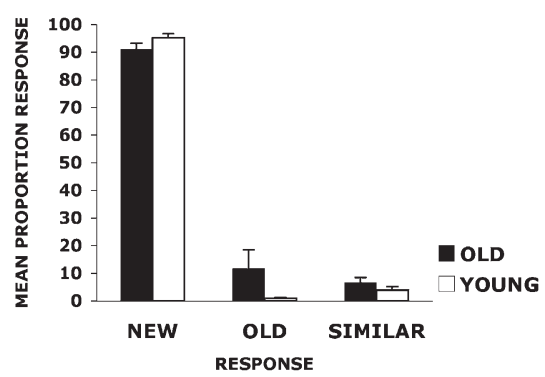

B

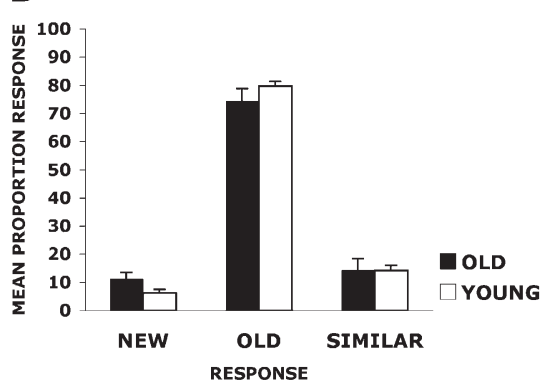

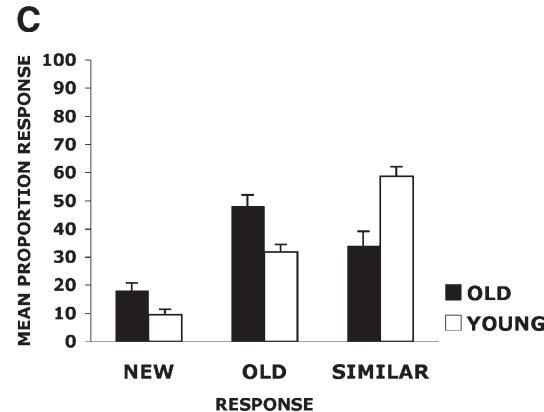

Figure 1. The mean proportion of responses (+ SE) of old, similar, or new to the (A) first presentation of objects (FIRST), $(B)$ objects presented previously during the experiment (REPEATED), and (C) objects that were similar to previously presented objects (LURE) for young and older adults on a continuous recognition memory task for visual objects. 


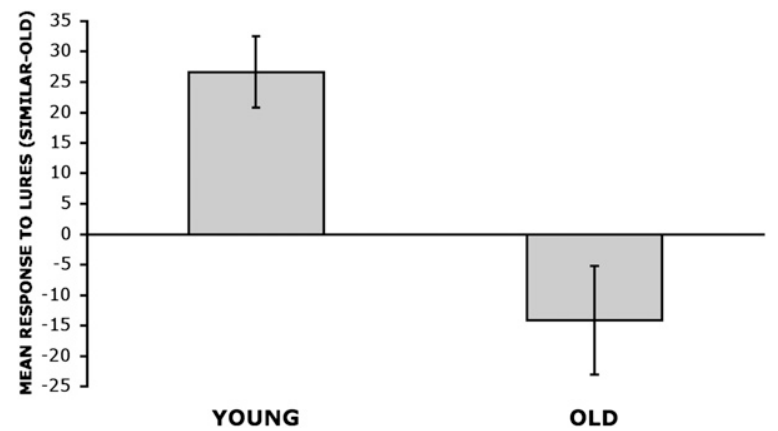

Figure 2. Mean difference scores (number of similar responses to lure stimuli - number of old responses to lure stimuli) for young and older adults on a continuous recognition memory task for visual objects. Lure objects were very similar but not identical to previously presented objects.

fiber projections to the CA3 subregion of the hippocampus. The DG may be particularly susceptible to age-related changes (Small et al. 2002, 2004). In addition, neuroimaging studies have demonstrated that the present task is associated with activation of the DG and CA3 subregions of the hippocampus and may be a good measure of pattern separation (Kirwan and Stark 2007; Bakker et al. 2008). A recent model published by Wilson et al. (2006) suggests that age-related changes in the DG may impair the ability to reduce similarity among new input patterns, resulting in decreased efficiency in pattern separation. In addition, the model proposes that, with advanced age, the CA3 subregion may become entrenched in pattern completion (Wilson et al. 2006). Pattern completion is described as a mechanism by which a pattern of activation can be completed or retrieved based on incomplete input (McNaughton and Nadel 1989; O'Reilly and Rudy 2000; Guzowski et al. 2004; Lee et al. 2004; Kesner et al. 2005; Rolls and Kesner 2006). The model proposes that age-related changes in the hippocampus strengthen the autoassociative network of the CA3 subregion at a cost of processing new information and enhancing pattern separation (Wilson et al. 2006). In the present task, when lure stimuli were presented, age-related changes in the DG may decrease pattern separation, whereas pattern completion may be increased in the CA3 subregion. Therefore, rather than correctly identifying a lure stimulus as similar based on the operation of a pattern separation mechanism, pattern completion may dominate and the lure stimulus may be incorrectly identified as an old stimulus, resulting in a false positive error.

To examine the number of correct responses to lure stimuli relative to the number of false positive responses to lures, difference scores were calculated for each participant. A positive difference score is indicative of more correct responses to lure stimuli than incorrect responses. Therefore, efficient pattern separation is reflected by higher difference scores in the positive direction. A negative mean difference score is indicative of more incorrect responses to lure stimuli than correct responses, suggesting decreased pattern separation efficiency and an increase in false positive errors. The average difference score for the younger adults suggests that they were more likely to label a lure stimulus as similar rather than old (Fig. 2). However, the average difference score for the older adults suggests that they were more likely to label a lure stimulus as old rather than similar. Thus, the present data are consistent with the model proposed by Wilson et al. (2006). The present findings suggest that pattern separation may be less efficient in nondemented older adults and the findings offer insight into a potential mechanism that may contribute to increased false positive errors.

Older adult participants also completed a battery of standardized neuropsychological tests including subscales from the
D-KEFS and the HVLT-R. Executive function tasks from the DKEFS are thought to be sensitive to frontal lobe function, whereas serial list learning tests such as the HVLT-R are thought to rely on medial temporal lobe function. Pearson $r$ correlations were used to examine relationships between the ability of older adults to correctly identify lure stimuli and performance on the measures from the HVLT-R and the D-KEFS (Table 1). The ability of older adults to correctly identify lures was significantly correlated with indices of medial temporal lobe function on the HVLT-R, with the exception of recognition discriminability. The executive function measures on the Trail Making Test (number-letter switching) or Color-Word Interference Test (inhibition and inhibition/switching) were not correlated with number of lures correctly identified. However, a significant correlation was detected between task performance and number sequencing, a basic component process involved in completing the number-letter switching executive function task. The results suggest a relationship between performance on the present visual pattern separation task and performance on standardized tests that are sensitive to medial temporal lobe function. However, there was no relationship between performance on the present task and executive function tests sensitive to frontal lobe function.

Although the aforementioned functional neuroimaging studies suggest that the human DG and CA3 hippocampal subregions support pattern separation based on overlapping object features (Kirwan and Stark 2007; Bakker et al. 2008), the perirhinal cortex also may play a role in pattern separation for objects. Lesion studies involving rats and monkeys indicate that lesions of the perirhinal cortex typically disrupt object recognition; however, hippocampal lesions produce variable results on object recognition tasks (for review, see Murray et al. 2000). Specifically, studies have shown that the perirhinal cortex may function to distinguish between visual objects with overlapping features to reduce feature ambiguity (Bussey et al. 2002, 2006; Gilbert and Kesner 2003; Norman and Eacott 2004; Bartko et al. 2007). However, some studies have suggested that the hippocampus may play little role is disambiguating object features (Saksida et al. 2006). Studies using stereological methods for quantifying neuronal numbers in rodents (Rapp et al. 2002) and magnetic resonance imaging in humans (Insausti et al. 1998) have reported that total neuron numbers and cortical volumes are largely preserved in the perirhinal cortex in aged rats and humans, respectively. Others have postulated that age-related functional changes in perirhinal cortex

Table 1. A summary of the results from Pearson $r$ correlations

\begin{tabular}{lll}
\hline HVLT-R & & \\
\hline Total immediate recall & $r=0.57$ & $P<0.01$ \\
Delayed recall & $r=0.64$ & $P<0.01$ \\
Retention & $r=0.56$ & $P<0.05$ \\
Recognition discriminability & $r=0.13$ & $P=0.59$ \\
D-KEFS trail making test & & \\
Visual scanning & $r=-0.13$ & $P=0.59$ \\
$\quad$ Number sequencing & $r=-0.35$ & $P=0.13$ \\
Letter sequencing & $r=-0.62$ & $P<0.01$ \\
Number-letter switching & $r=-0.17$ & $P=0.49$ \\
Motor speed & $r=-0.32$ & $P=0.16$ \\
D-KEFS Color-Word Interference Test & & \\
Color naming & $r=-0.12$ & $P=0.64$ \\
Word reading & $r=-0.12$ & $P=0.63$ \\
Inhibition & $r=-0.39$ & $P=0.09$ \\
Inhibition/switching & $r=-0.30$ & $P=0.21$
\end{tabular}

Summary of results examines the relationships between the ability of older adults to correctly identify lure stimuli and performance on standardized neuropsychological measures from the HVLT-R and the D-KEFS. 
may result in deficits on tasks such as fear conditioning in rodents (Moyer Jr. and Brown 2006). Therefore, age-related functional changes in the perirhinal cortex also may contribute to the decreased ability of older adults to identify lure stimuli as similar in the present study.

In conclusion, age-related changes in the hippocampal subregions of nondemented older adults may result in less efficient pattern separation. As a result, older adults may demonstrate poorer recognition memory performance when interference is increased. Pattern separation tasks have been shown to be particularly sensitive to DG function in both humans and animal models. Since the DG may be particularly sensitive to age-related changes in the hippocampus, the present task offers unique insight into the possible behavioral effects of age-related DG dysfunction. In addition, functional changes in the perirhinal cortex also may contribute to decreased visual object pattern separation in older adults. The present findings may have important implications for designing and evaluating standardized neuropsychological tests for assessing age-related changes in recognition memory. In addition, the identification of key mnemonic processing deficits in pattern separation may result in behavioral interventions for older adults that structure daily living tasks to mitigate interference, resulting in improved recognition memory. A current goal of Alzheimer's disease research is to identify early markers for disease onset during the very early stages and to differentiate these markers from those associated with normal aging. Since normal and pathological aging may have differential effects on the subregions of the hippocampus, tasks sensitive to dysfunction in particular subregions of the hippocampus, such as the DG, may help to differentiate cognitive impairment associated with normal aging from pathological changes associated with Alzheimer's disease.

\section{Acknowledgments}

This research was supported by a San Diego State University Grants Program Award to P.E.G. We thank Jerlyn Tolentino for assistance with participant recruitment and Shelley Jones, Peggy Ranke, Breeanna Bailey, and Adrienne Collazo for their assistance with data collection. We also thank Drs. Claire Murphy and Charles Degeneffe for their helpful suggestions on the study.

\section{References}

Allen, J.S., Bruss, J., Brown, C.K., and Damasio, H. 2005. Normal neuroanatomical variation due to age: The major lobes and a parcellation of the temporal region. Neurobiol. Aging 26: 12451260.

Bakker, A., Kirwan, C.B., Miller, M., and Stark, C.E. 2008. Pattern separation in the human hippocampal CA3 and dentate gyrus. Science 319: 1640-1642.

Bartko, S.J., Winters, B.D., Cowell, R.A., Saksida, L.M., and Bussey, T.J. 2007. Perirhinal cortex resolves feature ambiguity in configural object recognition and perceptual oddity tasks. Learn. Mem. 14: $821-832$.

Braak, H. and Braak, E. 1996. Development of Alzheimer-related neurofibrillary changes in the neocortex inversely recapitulates cortical myelogenesis. Acta Neuropathol. 92: 197-201.

Brandt, J. 1991. The Hopkins Verbal Learning Test: Development of a new memory test with six equivalent forms. Clin. Neuropsychol. 5: $125-142$.

Bussey, T.J., Saksida, L.M., and Murray, E.A. 2002. Perirhinal cortex resolves feature ambiguity in complex visual discriminations. Eur. J. Neurosci. 15: $365-374$.

Bussey, T.J., Saksida, L.M., and Murray, E.A. 2006. Perirhinal cortex and feature-ambiguous discriminations. Learn. Mem. 13: 103-105.

Cravens, C.J., Vargas-Pinto, N., Christian, K.M., and Nakazawa, K. 2006. CA3 NMDA receptors are crucial for rapid and automatic representation of context memory. Eur. J. Neurosci. 24: 1771-1780.

Delis, D., Kaplan, E., and Kramer, J.H. 2001. Delis-Kaplan Executive Function System (D-KEFS). The Psychological Corporation, San Antonio, TX.
Driscoll, I. and Sutherland, R.J. 2005. The aging hippocampus: Navigating between rat and human experiments. Rev. Neurosci. 16: 87-121.

Driscoll, I., Howard, S.R., Monfils, M.H., Tomanek, B., Brooks, W.M., and Sutherland, R.J. 2006. The aging hippocampus: A multi-level analysis in the rat. Neuroscience 139: 1173-1185.

Gilbert, P.E. and Kesner, R.P. 2003. Recognition memory for complex visual discriminations is affected by stimulus interference in rodents with perirhinal cortex damage. Learn. Mem. 10: 525-530.

Gilbert, P.E. and Kesner, R.P. 2006. The role of the dorsal CA3 hippocampal subregion in spatial working memory and pattern separation. Behav. Brain Res. 169: 142-149.

Gilbert, P.E., Kesner, R.P., and DeCoteau, W.E. 1998. Role of the hippocampus in mediating spatial pattern separation. J. Neurosci. 18: 804-810.

Gilbert, P.E., Kesner, R.P., and Lee, I. 2001. Dissociating hippocampal subregions: A double dissociation between dentate gyrus and CA1. Hippocampus 11: 626-636.

Good, C.D., Johnsrude, I.S., Ashburner, J., Henson, R.N., Friston, K.J., and Frackowiak, R.S. 2001. A voxel-based morphometric study of aging in 465 normal adult human brains. Neuroimage 14: 21-36.

Goodrich-Hunsaker, N.J., Hunsaker, M.R., and Kesner, R.P. 2008. The interactions and dissociations of the dorsal hippocampus subregions: How the dentate gyrus, CA3, and CA1 process spatial information. Behav. Neurosci. 122: 16-26.

Guzowski, J.F., Knierim, J.J., and Moser, E.I. 2004. Ensemble dynamics of hippocampal regions CA3 and CA1. Neuron 44: 581-584.

Hunsaker, M.R. and Kesner, R.P. 2008. Evaluating the temporal context of episodic memory: The role of CA3 and CA1. Behav. Brain Res. 188: 310-315.

Insausti, R., Juottonen, K., Soininen, H., Insausti, A.M., Partanen, K., Vainio, P., Laakso, M.P., and Pitkänen, A. 1998. MR volumetric analysis of the human entorhinal, perirhinal, and temporopolar cortices. AJNR Am. J. Neuroradiol. 19: 659-671.

Kesner, R.P. 2007. A behavioral analysis of dentate gyrus function. Prog. Brain Res. 163: 567-576.

Kesner, R.P., Hunsaker, M.R., and Gilbert, P.E. 2005. The role of CA1 in the acquisition of an object-trace-odor paired associate task. Behav. Neurosci. 119: $781-786$.

Kirwan, B.C. and Stark, C.E.L. 2007. Pattern separation processes in the human medial temporal lobe: An fMRI investigation. Learn. Mem. 14: 625-633.

Kubik, S., Miyashita, T., and Guzowski, J.F. 2007. Using immediate-early genes to map hippocampal subregional functions. Learn. Mem. 14: 758-770.

Lee, I., Roa, G., and Knierim, J.J. 2004. A double dissociation between hippocampal subfields: Differential time course of CA3 and CA1 place cells for processing changed environments. Nueron. 42: 803-815.

Lee, I., Jerman, T.S., and Kesner, R.P. 2005. Disruption of delayed memory for a sequence of spatial locations following CA1- or CA3-lesions of the dorsal hippocampus. Neurobiol. Learn. Mem. 84: 138-147.

Leutgeb, S. and Leutgeb, J.K. 2007. Pattern separation, pattern completion, and new neuronal codes within a continuous CA3 map. Learn. Mem. 14: 745-757.

Leutgeb, S., Leutgeb, J.K., Treves, A., Moser, M.B., and Moser, E.I. 2004. Distinct ensemble codes in hippocampal areas CA3 and CA1. Science 305: $1295-1298$.

Leutgeb, S., Leutgeb, J.K., Barnes, C.A., Moser, E.I., McNaughton, B.L., and Moser, M.B. 2005. Independent codes for spatial and episodic memory in hippocampal neuronal ensembles. Science 309: 619-623.

Leutgeb, J.K., Leutgeb, S., Moser, M.B., and Moser, E.I. 2007. Pattern separation in the dentate gyrus and CA3 of the hippocampus. Science 315: 961-966.

Marr, D. 1971. Simple memory: A theory for archicortex. Philos. Trans. $R$. Soc. Lond. B Biol. Sci. 262: 23-81.

Mattis, S. 1976. Mental status examination for organic mental syndrome in the elderly patient. In Geriatric psychiatry: A handbook for psychiatrists and primary care physicians (eds. L. Bellack and T.B. Katsau), pp. 77-121. Grune and Stratton, New York.

McHugh, T.J., Jones, M.W., Quinn, J.J., Balthasar, N., Coppari, R., Elmquist, J.K., Lowell, B.B., Fanselow, M.S., Wilson, M.A., and Tonegawa, S. 2007. Dentate gyrus NMDA receptors mediate rapid pattern separation in the hippocampal network. Science 317: 94-99.

McNaughton, B.L. and Nadel, L. 1989. Hebbian-Marr networks and the neurobiological representation of action in space. In Neuroscience and connectionist theory (eds. M.A. Gluck and D.E. Rumelhart), pp. 1-63. Lawrence Erlbaum Associates, Hillsdale, NJ.

Moyer Jr., J.R. and Brown, T.H. 2006. Impaired trace and contextual fear conditioning in aged rats. Behav. Neurosci. 120: 612-624.

Murray, E.A., Bussey, T.J., Hampton, R.R., and Saksida, L.M. 2000. The parahippocampal region and object identification. Ann. N. Y. Acad. Sci. 911: $166-174$. 
Myers, C.E. and Scharfman, H.E. 2008. A role for hilar cells in pattern separation in the dentate gyrus: A computational approach. Hippocampus 12: 325-340.

Norman, G. and Eacott, M.J. 2004. Impaired object recognition with increasing levels of feature ambiguity in rats with perirhinal cortex lesions. Behav. Brain Res. 148: 79-91.

O'Reilly, R.C. and McClelland, J.L. 1994. Hippocampal conjunctive encoding, storage, and recall: Avoiding a trade-off. Hippocampus 4: 661 682.

O'Reilly, R.C. and Rudy, J.W. 2000. Computational principles of learning in the neocortex and hippocampus. Hippocampus 10: 389-397.

Patrylo, P.R. and Williamson, A. 2007. The effects of aging on dentate circuitry and function. Prog. Brain Res. 163: 679-696.

Price, J.L., Ko, A.I., Wade, M.J., Tsou, S.K., McKeel, D.W., and Morris, J.C. 2001. Neuron number in the entorhinal cortex and CA1 in preclinical Alzheimer disease. Arch. Neurol. 58: 1395-1402.

Rapp, P.R., Deroche, P.S., Mao, Y., and Burwell, R.D. 2002. Neuron number in the parahippocampal region is preserved in aged rats with spatial learning deficits. Cereb. Cortex 12: 1171-1179.

Raz, N., Lindenberger, U., Rodrigue, K.M., Kennedy, K.M., Head, D., and Williamson, A. 2005. Regional brain changes in aging healthy adults: General trends, individual differences and modifiers. Cereb. Cortex 15: 1679-1689.

Renaudineau, S., Poucet, B., and Save, E. 2007. Flexible use of proximal objects and distal cues by hippocampal place cells. Hippocampus 17: 381-395.

Rolls, E. 1996. A theory of hippocampal function in memory. Hippocampus 6: $601-620$.

Rolls, E.T. 2007. An attractor network in the hippocampus: Theory and neurophysiology. Learn. Mem. 14: 714-731.

Rolls, E.T. and Kesner, R.P. 2006. A computational theory of hippocampal function, and empirical tests of the theory. Prog. Neurobiol. 79. $1-48$.
Rosenzweig, E.S. and Barnes, C.A. 2003. Impact of aging on hippocampal function: Plasticity, network dynamics, and cognition. Prog. Neurobiol. 69: 143-179.

Saksida, L.M., Bussey, T.J., Buckmaster, C.A., and Murray, E.A. 2006. No effect of hippocampal lesions on perirhinal cortex-dependent feature-ambiguous visual discriminations. Hippocampus 16: 421-430.

Shapiro, M.L. and Olton, D.S. 1994. Hippocampal function and interference. In Memory Systems 1994 (eds. D.L. Schacter and E. Tulving), pp. 141-146. MIT Press, London, UK.

Small, S.A., Tsai, W.Y., DeLaPaz, R., Mayeux, R., and Stern, Y. 2002. Imaging hippocampal function across the human life span: Is memory decline normal or not? Ann. Neurol. 51: 290-295.

Small, S.A., Chawla, M.K., Buonocore, M., Rapp, P.R., and Barnes, C.A. 2004. Imaging correlates of brain function in monkeys and rats isolates a hippocampal subregion differentially vulnerable to aging. Neuroscience 101: 7181-7186.

Tanila, H. 1999. Hippocampal place cells can develop distinct representations of two visually identical environments. Hippocampus 9: 235-246.

Walhovd, K., Fjell, A., Reinvang, I., Lundervold, A., Dale, A., Eilertsen, D. Quinn, B., Salat, D., Makris, N., and Fischl, B. 2005. Effects of age on volumes of cortex, white matter and subcortical structures. Neurobiol. Aging 26: 1261-1270.

West, M.J., Kawas, C.H., Martin, L.J., and Troncoso, J.C. 2001. The CA1 region of the human hippocampus is a hot spot in Alzheimer's disease. Ann. N.Y. Acad. Sci. 908: 255-259.

Wilson, I.A., Gallagher, M., Eichenbaum, H., and Tanila, H. 2006. Neurocognitive aging: Prior memories hinder new hippocampal encoding. Trends Neurosci. 29: 662-670.

Received December 19, 2008; accepted in revised form March 5, 2009. 


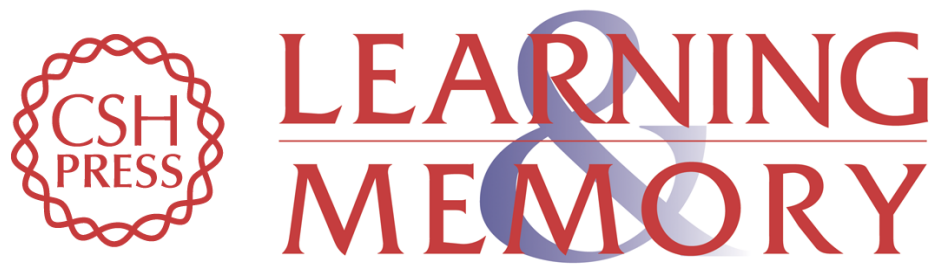

\section{Visual object pattern separation deficits in nondemented older adults}

Chelsea K. Toner, Eva Pirogovsky, C. Brock Kirwan, et al.

Learn. Mem. 2009, 16:

Access the most recent version at doi:10.1101//m.1315109

References This article cites 53 articles, 13 of which can be accessed free at:

http://learnmem.cshlp.org/content/16/5/338.full.html\#ref-list-1

License

Email Alerting Receive free email alerts when new articles cite this article - sign up in the box at the Service top right corner of the article or click here. 